\title{
ON THE HOMEOMORPHISMS OF THE SPACE OF GEODESIC LAMINATIONS ON A HYPERBOLIC SURFACE
}

\author{
C. CHARITOS, I. PAPADOPERAKIS, AND A. PAPADOPOULOS
}

(Communicated by Alexander N. Dranishnikov)

\begin{abstract}
We prove that for any orientable connected surface $S$ of finite type which is not a sphere with at most four punctures or a torus with at most two punctures, the natural homomorphism from the extended mapping class group of $S$ to the group of homeomorphisms of the space of geodesic laminations on $S$, equipped with the Thurston topology, is an isomorphism.
\end{abstract}

\section{INTRODUCTION}

In this paper $S=S_{g, p}$ is an orientable connected surface of finite type, of genus $g \geq 0$ with $p \geq 0$ punctures. We assume that $S$ is not a sphere with at most four punctures or a torus with at most two punctures. Fixing a complete hyperbolic metric of finite area on $S$, we consider the set $\mathcal{G} \mathcal{L}(S)$ of geodesic laminations on $S$ with compact support. The set $\mathcal{G} \mathcal{L}(S)$, as a set of compact subspaces of the metric space $S$, is equipped with the Hausdorff metric, which we denote by $d_{H}$. We denote by $\mathcal{T}_{H}$ the topology induced by $d_{H}$, and we call it the Hausdorff topology. We consider on $\mathcal{G} \mathcal{L}(S)$ a second topology $\mathcal{T}$, referred to as the Thurston topology (see the definition in $₫ 2$ below) and which is weaker than $\mathcal{T}_{H}$. The space $\mathcal{G L}(S)$, equipped with the Thurston topology, is not as well known as other spaces of laminations, e.g. the space of measured laminations, but it plays a major role in Thurston's theory of Kleinian groups; see [12, Chapter 8, in particular §8.1. Any homeomorphism $h: S \rightarrow S$ induces by push-forward a map $h_{*}: \mathcal{G L}(S) \rightarrow \mathcal{G L}(S)$ which is a homeomorphism with respect to both topologies, $\mathcal{T}_{H}$ and $\mathcal{T}$. The main result of this paper is the following:

Theorem 1.1. The natural homomorphism from the extended mapping class group of $S$ to the group of homeomorphisms of $\mathcal{G} \mathcal{L}(S)$ equipped with the Thurston topology is an isomorphism.

In particular, if $f: \mathcal{G L}(S) \rightarrow \mathcal{G} \mathcal{L}(S)$ is a homeomorphism with respect to the topology $\mathcal{T}$, then there is a homeomorphism $h: S \rightarrow S$ such that $h_{*}=f$.

This shows that the space $\mathcal{G} \mathcal{L}(S)$ equipped with the Thurston topology is definitely not topologically homogeneous. Furthermore, since its homeomorphism group is countable, this space does not contain any open set which is a manifold of positive dimension, in contrast with measured lamination space which is a manifold (topologically, it is a sphere).

Received by the editors December 26, 2011 and, in revised form, July 3, 2012 and July 9, 2012. 2010 Mathematics Subject Classification. Primary 57M50; Secondary 20F65, 57R30.

Key words and phrases. Geodesic lamination, mapping class group, hyperbolic surface, Hausdorff topology, Thurston topology. 
The analogous result for the Hausdorff topology is not true as simple closed geodesics are all isolated points, and therefore we can find homeomorphisms of this space that are not induced by surface homeomorphisms.

The result of Theorem 1.1 is in the spirit of several rigidity results that were obtained by various authors in the context of mapping class group actions on different spaces, and it is especially close to the results in [11, 8 and 9 which concern actions by homeomorphisms. We note that although the statements of these and other rigidity theorems look alike, all of them are interesting, because each proof displays new features of the space on which the mapping class group acts in different settings (combinatorial, topological, holomorphic, metric, etc.), and the arguments in each setting are often different.

The proof of Theorem 1.1 is given in 4 . It involves the construction, from the homeomorphism $f$, of an automorphism of the complex of curves of $S$. The main technical point is to show that $f$ preserves the set of finite laminations. For the passage between properties of laminations and homeomorphisms of $\mathcal{G} \mathcal{L}(S)$, the idea is to translate inclusions $\Lambda_{1} \subset \Lambda_{2}$ between geodesic laminations into set-theoretical properties of open sets of $\mathcal{G} \mathcal{L}(S)$.

\section{DEFINITIONS AND PRELIMINARIES}

On the surface $S$, we fix a complete hyperbolic metric of finite area. A geodesic lamination $\Lambda \subset S$ is a compact non-empty subset which is the union of disjoint simple geodesics. We say that a geodesic lamination is maximal if it is not a proper sublamination of any other geodesic lamination. We say that a geodesic lamination is minimal if every leaf of the lamination is dense in the support of the lamination. It follows that a lamination is minimal if and only if it does not contain a proper sublamination.

A geodesic lamination which is a finite union of geodesics is called a finite lamination. Otherwise, it is said to be infinite.

The following two subsets of $\mathcal{G L}(S)$ will play special roles in the sequel:

- $\mathcal{F} \mathcal{G} \mathcal{L}(S)$ is the subset of finite laminations of $\mathcal{G L}(S)$. An element of $\mathcal{F} \mathcal{G} \mathcal{L}(S)$ is made out of a finite union of disjoint simple closed geodesics $\left\{\gamma_{i}\right\}$ together with a finite number of infinite geodesics, each spiraling from each end around one geodesic in $\left\{\gamma_{i}\right\}$.

- $\mathcal{C G} \mathcal{L}(S)$ is the set of geodesic laminations whose leaves are simple closed geodesics.

Obviously $\mathcal{C G} \mathcal{L}(S) \subset \mathcal{F} \mathcal{G} \mathcal{L}(S)$.

For any subset $X$ of $S$ and for any $\epsilon>0$, we set

$$
N_{\varepsilon}(X)=\{x \in S: \exists y \in X \text { with } d(x, y)<\varepsilon\} .
$$

The following definition is classical:

Definition 2.1. Let $X$ and $X^{\prime}$ be two compact subsets of $S$. The Hausdorff distance between $X$ and $X^{\prime}$ is the quantity

$$
d_{H}\left(X, X^{\prime}\right)=\inf \left\{\varepsilon>0: X \subset N_{\varepsilon}\left(X^{\prime}\right) \text { and } X^{\prime} \subset N_{\varepsilon}(X\} .\right.
$$

It is easy to see that the function $d_{H}$ is a distance function on the set of compact subsets of $S$. Such a definition was made by F. Hausdorff for the set of compact subsets of $\mathbb{R}^{n}$, and it was used by H. Busemann [1] for the set of compact sets of a general metric space. 
We shall mostly use the notions $N_{\varepsilon}(X)$ and $d_{H}(X, Y)$ for elements $X, Y \subset S$ which are geodesic laminations on $S$. We also denote by $d_{H}$ the restriction of the Hausdorff metric to $\mathcal{G} \mathcal{L}(S)$.

We also use the following notation:

For any $\Lambda \in \mathcal{G} \mathcal{L}(S)$ and for any $\varepsilon>0$,

$$
\mathcal{V}_{\varepsilon}(\Lambda)=\left\{\Lambda^{\prime} \in \mathcal{G} \mathcal{L}(S): N_{\varepsilon}\left(\Lambda^{\prime}\right) \supset \Lambda \text { and } N_{\varepsilon}(\Lambda) \supset \Lambda^{\prime}\right\} \text {. }
$$

The topology induced by $d_{H}$ on the set of subsets of $S$ as well as its restriction to $\mathcal{G} \mathcal{L}(S)$, which we shall denote by $\mathcal{T}_{H}$, is called the Hausdorff topology. For the topology $\mathcal{T}_{H}$ any set $\mathcal{V}_{\varepsilon}(\Lambda)$ is open. Moreover, it is easy to see that the collection of sets $\mathcal{V}_{\varepsilon}(\Lambda)$ for all $\varepsilon>0$ and $\Lambda \in \mathcal{G} \mathcal{L}(S)$ constitute a basis for $\mathcal{T}_{H}$.

We now equip the set $\mathcal{G} \mathcal{L}(S)$ with a second topology.

Definition 2.2. Let $V$ be an open subset of $S$. Set

$$
\mathcal{U}_{V}=\{\Lambda \in \mathcal{G} \mathcal{L}(S): \Lambda \cap V \neq \emptyset\} .
$$

We let $\mathcal{T}$ be the topology on $\mathcal{G} \mathcal{L}(S)$ with subbasis the sets $\mathcal{U}_{V}$, where $V$ varies over the set of open subsets of $S$.

Following the terminology of ([2], Def. I.4.1.10), we call the topology $\mathcal{T}$ on $\mathcal{G} \mathcal{L}(S)$ the Thurston topology. The original reference for the topology $\mathcal{T}$ is (Thurston [12], Section 8.10), where $\mathcal{T}$ is referred to as the geometric topology. Clearly the topology $\mathcal{T}$ does not satisfy the first axiom of separation. Indeed, take a geodesic lamination $\Lambda$ that contains a strict sublamination $\Lambda_{1} \varsubsetneqq \Lambda$; then every open set for $\mathcal{T}$ containing $\Lambda_{1}$ contains $\Lambda$. In particular the topology $\mathcal{T}$ is not Hausdorff, unlike the topology $\mathcal{T}_{H}$, which is induced by a metric. Thurston describes in [12] the topology $\mathcal{T}$ in terms of train track (without weights) approximations and uses the space $\mathcal{G} \mathcal{L}(S)$, equipped with this topology, in his theory of realizing geodesic laminations in 3manifolds using maps from hyperbolic surfaces to 3-manifolds which send cusps to cusps.

Lemma 2.3. $\mathcal{T} \subset \mathcal{T}_{H}$; i.e. the topology $\mathcal{T}$ is weaker than the topology $\mathcal{T}_{H}$.

Proof. It suffices to prove that for each open subset $V$ of $S, \mathcal{U}_{V} \in \mathcal{T}_{H}$. For $\Lambda \in \mathcal{U}_{V}$, we have $\Lambda \cap V \neq \emptyset$. For any $x \in \Lambda \cap V$, there exists an open ball $B\left(x, \varepsilon_{\Lambda}\right)$ in $S$ of center $x$ and radius $\varepsilon_{\Lambda}$ such that $B\left(x, \varepsilon_{\Lambda}\right) \subset V$. We now prove the following:

$$
\Lambda \in \mathcal{V}_{\varepsilon_{\Lambda}}(\Lambda) \subset \mathcal{U}_{V}
$$

First, it is obvious that $\Lambda \in \mathcal{V}_{\varepsilon_{\Lambda}}(\Lambda)$. To prove the inclusion $\mathcal{V}_{\varepsilon_{\Lambda}}(\Lambda) \subset \mathcal{U}_{V}$ we note that if $\Lambda^{\prime} \in \mathcal{V}_{\varepsilon_{\Lambda}}(\Lambda)$, then $\Lambda \subset N_{\varepsilon_{\Lambda}}\left(\Lambda^{\prime}\right)$, and hence $x \in N_{\varepsilon_{\Lambda}}\left(\Lambda^{\prime}\right)$. Therefore we can find a point $y$ in $\Lambda^{\prime}$ such that $d(x, y)<\varepsilon_{\Lambda}$, and hence $y \in \Lambda^{\prime} \cap B\left(x, \varepsilon_{\Lambda}\right) \neq \emptyset$. Since $B\left(x, \varepsilon_{\Lambda}\right) \subset V$, this implies that $\Lambda^{\prime} \cap V \neq \emptyset$. Therefore $\Lambda^{\prime} \in \mathcal{U}_{V}$ and the inclusion $\mathcal{V}_{\varepsilon_{\Lambda}}(\Lambda) \subset \mathcal{U}_{V}$ is proven.

Now from (1) we deduce immediately that

$$
\mathcal{U}_{V}=\bigcup\left\{\mathcal{V}_{\varepsilon_{\Lambda}}(\Lambda): \Lambda \in \mathcal{G} \mathcal{L}(S) \text { and } \Lambda \cap V \neq \emptyset\right\} .
$$

Therefore $\mathcal{U}_{V} \in \mathcal{T}_{H}$.

The metric space $\left(\mathcal{G L}(S), d_{H}\right)$ is compact. This is a general result of the Hausdorff metric on the set $\mathcal{B}(X)$ of compact subsets of a metric space $X$, and $\mathcal{G} \mathcal{L}(S)$ is a closed subset of $\mathcal{B}(S)$ (cf. [3], Theorem 3.4, for this special case). Therefore $\mathcal{T}_{H}$ is a compact topology. Since $\mathcal{T} \subset \mathcal{T}_{H}$, it follows that $\mathcal{T}$ is also a compact topology. 
In the next theorem we summarize basic properties of minimal geodesic laminations and we also give a description of the structure of maximal geodesic laminations. The properties are all well known from Thurston's theory, and we give references for the convenience of the reader.

Theorem 2.4. (I) (Proposition A.2.1, p. 142 in [10]). Let $\Lambda$ be an arbitrary geodesic lamination of $S$. Then $S-\Lambda$ consists of finitely many components. Let $U$ be such a component. The completion $C(U)$ of $U$ with respect to the metric induced by the Riemannian metric of $S$ is a complete hyperbolic surface of finite area with geodesic boundary.

(II) (Corollary A.2.4, p. 143 in [10] or Lemmata 4.2 and 4.3 in [3]). Let $\Lambda$ be a minimal geodesic lamination with infinitely many leaves. Then every leaf of $\Lambda$ is dense in $\Lambda$. Furthermore, $\Lambda$ contains a finite number of leaves which are isolated from one side. These leaves appear as boundary geodesics of some $C(U)$, where $U$ is a component of $S-\Lambda$; they will be referred to as boundary leaves of $\Lambda$.

(III) (Theorem I.4.2.8, p. 83 in [2]). Let $\Lambda$ be an arbitrary geodesic lamination of $S$. Then $\Lambda$ consists of the disjoint union of a finite number of minimal sublaminations of $\Lambda$ together with a finite set of additional geodesics, each end of which spirals onto a minimal lamination. Each of the additional geodesics is isolated; i.e. it is contained in an open subset of $S$ which is disjoint from the rest of the lamination.

In item (III) above, the fact that an end of a geodesic spirals on a minimal sublamination $\Lambda^{\prime}$ of $\Lambda$ means that the set of accumulation points of this end on the surface is $\Lambda^{\prime}$.

The following lemma and proposition will also be used in the next section.

Lemma 2.5. Let $\Lambda$ be an infinite minimal geodesic lamination of $S$. Then $\Lambda$ has at least two boundary leaves.

Proof. Since the lamination $\Lambda$ is infinite and minimal, every boundary leaf is isolated on one side only (i.e. it is not an isolated leaf). Therefore, there is a bijection between the geodesics in the boundary of the completion of the complement of $\Lambda$ and the boundary leaves of this lamination (this uses the fact that there are no isolated leaves). The rest of the proof is by contradiction. Suppose there is a single (bi-infinite) geodesic in the boundary of the completion of the complement of $\Lambda$. Then by the Gauss-Bonnet Theorem, the area of the complement of $\Lambda$ would be an odd multiple of $\pi$. However, the area of this complement is equal to the area of the original surface, which is an even multiple of $\pi$. This contradiction proves the lemma.

Proposition 2.6 (see [12, Proposition 8.10.7, and [2], I 4.2.14, p. 81). The finite laminations are dense in the space of geodesic laminations equipped with the Hausdorff topology. Hence the same holds for the Thurston topology.

\section{On the ACtion of homeOMORPhisms of $\mathcal{G} \mathcal{L}(S)$}

We denote by $\mathcal{O}(S)$ the set of open subsets of $S$ and we fix an element $\Lambda$ of $\mathcal{G} \mathcal{L}(S)$. We consider the sets

$$
\mathcal{O}_{\Lambda}(S)=\{V \in \mathcal{O}(S): V \cap \Lambda \neq \emptyset\} \subset \mathcal{O}(S)
$$

and

$$
\mathcal{U}(\Lambda)=\bigcap_{V \in \mathcal{O}_{\Lambda}(S)} \mathcal{U}_{V} \subset \mathcal{G} \mathcal{L}(S)
$$


Since $\mathcal{U}(\Lambda)$ is the intersection of all subbasis elements containing $\Lambda$, it follows that $\mathcal{U}(\Lambda)$ is also the intersection of all open sets in $\mathcal{G} \mathcal{L}(S)$ containing $\Lambda$.

We have the following.

Lemma 3.1. Let $\Lambda_{1}, \Lambda_{2} \in \mathcal{G} \mathcal{L}(S)$. Then $\Lambda_{1} \subset \Lambda_{2}$ if and only if $\Lambda_{2} \in \mathcal{U}\left(\Lambda_{1}\right)$.

Proof. Assume that $\Lambda_{1} \subset \Lambda_{2}$. For any $V \in \mathcal{O}_{\Lambda_{1}}(S)$, we consider the set $\mathcal{U}_{V}$. Then $\Lambda_{2} \cap V \neq \emptyset$; hence $\Lambda_{2} \in \mathcal{U}_{V}$. Therefore $\Lambda_{2} \in \mathcal{U}\left(\Lambda_{1}\right)$.

Conversely, assume $\Lambda_{2} \in \mathcal{U}\left(\Lambda_{1}\right)$. If $\Lambda_{1}$ is not a subset of $\Lambda_{2}$, then there exists $x \in \Lambda_{1}$ with $x \notin \Lambda_{2}$. Since $\Lambda_{2}$ is a compact subset of $S$, there exists $\varepsilon>0$ such that the open ball $B(x, \varepsilon)$ does not intersect $\Lambda_{2}$. This implies that $\Lambda_{2} \notin \mathcal{U}_{B(x, \varepsilon)}$. This is a contradiction since $\Lambda_{2} \in \mathcal{U}\left(\Lambda_{1}\right)$. Hence $\Lambda_{1} \subset \Lambda_{2}$.

Lemma 3.2. Let $\Lambda_{1}, \Lambda_{2} \in \mathcal{G} \mathcal{L}(S)$. Then $\Lambda_{1} \subset \Lambda_{2}$ if and only if $\mathcal{U}\left(\Lambda_{2}\right) \subset \mathcal{U}\left(\Lambda_{1}\right)$.

Proof. First assume that $\Lambda_{1} \subset \Lambda_{2}$ and let $\Lambda^{\prime} \in \mathcal{U}\left(\Lambda_{2}\right)$. Then $\Lambda^{\prime} \cap V \neq \emptyset$ for each $V \in \mathcal{O}(S)$ with $V \cap \Lambda_{2} \neq \emptyset$. Therefore $\Lambda^{\prime} \cap V \neq \emptyset$ for each $V \in \mathcal{O}(S)$ with $V \cap \Lambda_{1} \neq \emptyset$. Therefore $\Lambda^{\prime} \in \mathcal{U}\left(\Lambda_{1}\right)$.

Now assume that $\mathcal{U}\left(\Lambda_{2}\right) \subset \mathcal{U}\left(\Lambda_{1}\right)$. Obviously, $\Lambda_{2} \in \mathcal{U}\left(\Lambda_{2}\right)$ and hence $\Lambda_{2} \in \mathcal{U}\left(\Lambda_{1}\right)$. From Lemma 3.1 this implies that $\Lambda_{1} \subset \Lambda_{2}$.

Lemma 3.3. Assume that $f$ is a homeomorphism of $\mathcal{G L}(S)$ with respect to the Thurston topology. If $\Lambda \in \mathcal{G} \mathcal{L}(S)$, then $f(\mathcal{U}(\Lambda))=\mathcal{U}(f(\Lambda))$.

Proof. Since $f$ is a homeomorphism, the open sets containing $f(\Lambda)$ are exactly the $f$-images of the open sets containing $\Lambda$ and hence the proof follows.

From the above lemmata, we obtain the following:

Corollary 3.4. Assume that $f$ is a homeomorphism of $\mathcal{G} \mathcal{L}(S)$ with respect to the Thurston topology and let $\Lambda_{1}, \Lambda_{2} \in \mathcal{G} \mathcal{L}(S)$. Then $\Lambda_{1} \subset \Lambda_{2}$ implies that $f\left(\Lambda_{1}\right) \subset$ $f\left(\Lambda_{2}\right)$.

Proof. From Lemma 3.2, the inclusion $\Lambda_{1} \subset \Lambda_{2}$ implies that $\mathcal{U}\left(\Lambda_{2}\right) \subset \mathcal{U}\left(\Lambda_{1}\right)$. Since $f$ is a bijection, we have $f\left(\mathcal{U}\left(\Lambda_{2}\right)\right) \subset f\left(\mathcal{U}\left(\Lambda_{1}\right)\right)$. From Lemma 3.3 we get $\mathcal{U}\left(f\left(\Lambda_{2}\right)\right) \subset$ $\mathcal{U}\left(f\left(\Lambda_{1}\right)\right)$, which implies again, by Lemma 3.2, that $f\left(\Lambda_{1}\right) \subset f\left(\Lambda_{2}\right)$.

Lemma 3.5. Let $f$ be a homeomorphism of $\mathcal{G} \mathcal{L}(S)$ with respect to the Thurston topology. Then $f$ sends a maximal (respectively minimal) geodesic lamination of $S$ to a maximal (respectively minimal) geodesic lamination of $S$.

Proof. Let $\Lambda$ be a maximal geodesic lamination of $S$. If $f(\Lambda)$ is not maximal, then there is a maximal geodesic lamination $\Theta$ such that $f(\Lambda) \varsubsetneqq \Theta$. Let $\Lambda^{\prime}=f^{-1}(\Theta)$. Then, by Corollary 3.4 we have $\Lambda \varsubsetneqq \Lambda^{\prime}$, which contradicts the maximality of $\Lambda$.

Likewise, let $\Lambda$ be a minimal geodesic lamination of $S$. If $f(\Lambda)$ is not minimal, then there exists a lamination $\Theta$ such that $\Theta \varsubsetneqq f(\Lambda)$. Let $\Lambda^{\prime}=f^{-1}(\Theta)$. Then, from Corollary 3.4 again, we have $\Lambda^{\prime} \varsubsetneqq \Lambda$, which contradicts the minimality of $\Lambda$.

From Theorem 2.4(III), every $\Lambda \in \mathcal{G L}(S)$ has a finite number of sublaminations. Thus, we give the following definition. 
Definition 3.6. Let $\Lambda \in \mathcal{G} \mathcal{L}(S)$. A chain of sublaminations of $\Lambda$ is a finite sequence $\left(\Lambda_{i}\right), i=0,1, \ldots, n$, of sublaminations of $\Lambda$ such that $\emptyset \neq \Lambda_{n} \varsubsetneqq \Lambda_{n-1} \varsubsetneqq \ldots \varsubsetneqq \Lambda_{1} \varsubsetneqq$ $\Lambda_{0}=\Lambda$. We denote such a chain by $\mathcal{C}_{\Lambda}$. The integer $n$ will be called the length of $\mathcal{C}_{\Lambda}$ and will be denoted by $l\left(\mathcal{C}_{\Lambda}\right)$.

A chain of sublaminations $\mathcal{C}_{\Lambda}$ will be called maximal if its length is maximal among all chains of sublaminations of $\Lambda$.

The length of a maximal chain of sublaminations $\mathcal{C}_{\Lambda}$ of $\Lambda$ depends only on $\Lambda$. Therefore the number $l\left(\mathcal{C}_{\Lambda}\right)$ will be referred to as the length of $\Lambda$ and will be denoted by length $(\Lambda)$.

Lemma 3.7. Let $f$ be a homeomorphism of $\mathcal{G} \mathcal{L}(S)$ with respect to the Thurston topology and let $\Lambda_{n} \varsubsetneqq \Lambda_{n-1} \varsubsetneqq \ldots \varsubsetneqq \Lambda_{1} \varsubsetneqq \Lambda_{0}=\Lambda$ be a maximal chain of sublaminations of $\Lambda$. Then $f\left(\Lambda_{n}\right) \varsubsetneqq f\left(\Lambda_{n-1}\right) \varsubsetneqq \ldots \varsubsetneqq f\left(\Lambda_{1}\right) \varsubsetneqq f\left(\Lambda_{0}\right)=f(\Lambda)$ is a maximal chain of sublaminations of $f(\Lambda)$ and length $\left(\Lambda_{k}\right)=\operatorname{length}\left(f\left(\Lambda_{k}\right)\right)$ for each $k=0,1, \ldots, n$.

Proof. The proof follows immediately from Corollary 3.4

We call a generalized pair of pants a hyperbolic surface which is homeomorphic to a sphere with three holes, a hole being either a geodesic boundary component or a cusp.

Now we can prove the following proposition.

Proposition 3.8. Let $f$ be a homeomorphism of $\mathcal{G} \mathcal{L}(S)$ with respect to the Thurston topology. Then,

(1) $f$ sends any maximal finite lamination which contains a collection of curves that decompose $S$ into a generalized pair of pants to a maximal finite lamination that has the same property.

(2) $f$ sends any laminations whose leaves are all closed to a lamination having the same property. Furthermore if such a lamination $\Lambda$ has $k$ components, then $f(\Lambda)$ also has $k$ components.

(3) $f$ sends finite laminations to finite laminations.

Proof. (1) Let $\Lambda$ be a maximal geodesic lamination. From Theorem 2.4 $S-\Lambda$ consists of finitely many open components.

Claim 1. Let $U$ be a component of $S-\Lambda$. Then, the completion $C(U)$ of $U$ is a hyperbolic surface which is isometric either to a hyperbolic ideal triangle or to a surface of genus 0 with a cusp and an open geodesic as its boundary. The latter will be referred to as a cusped hyperbolic monogon; it is obtained from a hyperbolic ideal triangle by gluing together two sides of this ideal triangle.

Proof of Claim 1. The surface $C(U)$ is a complete hyperbolic surface of finite area. Therefore $C(U)$ has finitely many boundary components which are either closed geodesics or open geodesics. We may easily verify that if $C(U)$ is not of the type described in the claim, then the lamination $\Lambda$ would not be maximal, because we could add open geodesics $l_{i}$ to $\Lambda$ and construct a lamination $\Lambda^{\prime} \supsetneqq \Lambda$. This proves Claim 1. 
Now let $\lambda_{1}, \ldots, \lambda_{n}$ be the leaves of $\Lambda$ which are boundary geodesics of the completion $C(U)$ of some component $U$ of $S-\Lambda$. The leaves $\lambda_{i}$ can be of two types:

(i) an open geodesic of $S$ which is an isolated leaf of $\Lambda$;

(ii) an open geodesic of $S$ which is a leaf isolated from one side in $\Lambda$.

A geodesic of type (ii) appears as a boundary leaf of a minimal infinite sublamination of $\Lambda$. Among the $\left\{\lambda_{i}\right\}$, we may assume, without loss of generality, that $\lambda_{1}, \ldots, \lambda_{k}$ are the isolated leaves of $\Lambda$, for $0 \leq k \leq n$. From Theorem 2.4 it follows that if $\Lambda$ is a finite maximal geodesic lamination, then $k=n$ and that if $\Lambda$ is a maximal infinite geodesic lamination, which is also minimal, then $k=0$.

To the leaves $\lambda_{1}, \ldots, \lambda_{n}$ of $\Lambda$ we add the leaves $c_{1}, \ldots, c_{m}$ of $\Lambda$ which are simple closed geodesics, if any exist. Let $A_{\Lambda}=\left\{\lambda_{1}, \ldots, \lambda_{n}, c_{1}, \ldots, c_{m}\right\}$.

We define a generating set for a geodesic lamination $\Lambda$ to be a set $\mathcal{A}=\left\{\mu_{1}, \ldots, \mu_{k}\right\}$ of leaves of $\Lambda$ such that the union of the closures of the leaves that belong to $\mathcal{A}$ is the lamination $\Lambda$. It follows from Theorem 2.4 that every geodesic lamination on $S$ has a finite generating set.

Our terminology is motivated by Claim 2, which follows now.

Claim 2. The set $A_{\Lambda}=\left\{\lambda_{1}, \ldots, \lambda_{n}, c_{1}, \ldots, c_{m}\right\}$ is a generating set for $\Lambda$, and any proper sublamination of $\Lambda$ is the union of closures of leaves that belong to some proper subset of $A_{\Lambda}$.

Proof of Claim 2. The claim follows immediately from the definition of $A$ and from Theorem 2.4 (III).

It is well known and easy to see, using an Euler characteristic count, that the maximum number of pairwise disjoint simple closed geodesics in $S$ is equal to $3 g-$ $3+b$ and that these geodesics cut $S$ into $2 g-2+b$ hyperbolic generalized pairs of pants. It is also easy to see that the maximum number of open geodesics $l_{i}$ of $S$ which decompose $S$ into hyperbolic ideal triangles is equal to $6 g-6+3 b$.

Claim 3. If $\Lambda$ is a maximal finite geodesic lamination that contains a generalized pair of pants decomposition $P$, then a maximal chain of sublaminations of $\Lambda$ has length $9 g-9+3 b$.

Proof of Claim 3. The lamination $\Lambda$ contains $3 g-3+b$ simple closed geodesics, say $c_{1}, \ldots, c_{3 g-3+b}$, which cut $S$ into generalized pairs of pants, and additional open isolated geodesics, say $\lambda_{1}, \ldots, \lambda_{r}$, such that each $\lambda_{i}$ spirals about some $c_{j}$. We may add to $\lambda_{i}$ open geodesics $\lambda_{k}^{\prime}$, which from one direction abut to a cusp and from the other direction spiral about some $c_{j}$, such that all the geodesics $\lambda_{i}$ and $\lambda_{i}^{\prime}$ decompose $S$ into hyperbolic ideal triangles. Since the total number of $\lambda_{i}$ and $\lambda_{i}^{\prime}$ is equal to $6 g-6+3 b$, we deduce that the number $r$ of $\lambda_{i}$ is equal to $6 g-6+2 b$. Therefore the set $A_{\Lambda}=\left\{\lambda_{1}, \ldots, \lambda_{6 g-6+2 b}, c_{1}, \ldots, c_{3 g-3+b}\right\}$ is a generating set of $\Lambda$. From Claim 2, a chain of sublaminations $\Lambda_{n} \varsubsetneqq \Lambda_{n-1} \varsubsetneqq \ldots \varsubsetneqq \Lambda_{1} \varsubsetneqq \Lambda_{0}=\Lambda$ is maximal if and only if for each $k=1, \ldots, n, \Lambda_{k}-\Lambda_{k-1}$ is a single leaf belonging to $A_{\Lambda}$. Such a sequence $\Lambda_{i}$ can be constructed as follows: From $\Lambda_{0}=\Lambda$ we first remove, one by one, all leaves $\lambda_{i}$; after removing all these leaves we continue removing, one by one, all leaves $c_{j}$. Obviously $n=9 g-9+3 b$.

Claim 4 . Let $\Lambda$ be a maximal finite geodesic lamination which does not contain a generalized pants decomposition. Then length $(\Lambda)<9 g-9+3 b$.

Proof of Claim 4. Since $\Lambda$ does not contain a generalized pants decomposition, it follows that if $c_{1}, \ldots, c_{k}$ are the closed geodesics in $\Lambda$, then $k$ is strictly smaller than 
$3 g-3+b$. Now as in the proof of Claim 3, we may prove that $\Lambda$ contains additional open geodesics $\lambda_{1}, \ldots, \lambda_{6 g-6+2 b}$ which spiral about the $c_{j}$ 's. Obviously, a maximal chain $\mathcal{C}_{\Lambda}$ of sublaminations of $\Lambda$ has length less than $9 g-9+3 b$.

Claim 5. Let $\Lambda$ be a maximal infinite geodesic lamination. Then length $(\Lambda)<$ $9 g-9+3 b$.

Proof of Claim 5. Consider a maximal chain of sublaminations of $\Lambda$, say $\Lambda_{k} \varsubsetneqq$ $\Lambda_{k-1} \varsubsetneqq \ldots \varsubsetneqq \Lambda_{1} \varsubsetneqq \Lambda_{0}=\Lambda$. Let $A_{\Lambda}=\left\{\lambda_{1}, \ldots, \lambda_{n}, c_{1}, \ldots, c_{m}\right\}$ be a generating set of $\Lambda$, where each $\lambda_{i}$ is an open geodesic and each $c_{i}$ is a closed geodesic, and we assume that the generating set $A_{\Lambda}$ is minimal in the sense that no proper subset of $A_{\Lambda}$ is a generating set of $\Lambda$.

First, we prove that $m<3 g-3+b$. We know that $m \leq 3 g-3+b$. If $m=3 g-3+b$, then the closed geodesics cut $S$ into generalized pairs of pants. This implies that a minimal sublamination, say $\Lambda^{\prime}$, of $\Lambda$ with infinitely may leaves must be in the interior of a generalized pair of pants. But it is easy to see that such a sublamination $\Lambda^{\prime}$ does not exist. Therefore $m<3 g-3+b$.

Second, we prove that $n \leq 6 g-6+2 b$. By Theorem 2.4, the leaves $\lambda_{1}, \ldots, \lambda_{n}$ are either isolated open leaves or boundary leaves. Since $\Lambda$ is maximal, for each component $U$ of $S-\Lambda$ the completion $C(U)$ is either a hyperbolic ideal triangle or a cusped hyperbolic monogon. The area of every such surface is equal to $\pi$; therefore, from the Gauss-Bonnet theorem the number of components $U$ is equal to $4 g-4+2 b$. Now, each hyperbolic ideal triangle has three sides and each cusped hyperbolic monogon has one side. On the other hand the number of cusped hyperbolic monogons is equal to $b$. Therefore the total number of sides of $C(U)$ is $3(4 g-4+2 b)-2 b=12 g-12+4 b$. Now if a leaf $\lambda_{i}$ is isolated it belongs exactly to two components $C(U)$. If a leaf $\lambda_{i}$ is not isolated, then it belongs to a minimal sublamination of $\Lambda$, say $K_{i}$. From Lemma 2.5] $K_{i}$ contains at least two boundary leaves, and we take $\lambda_{i}$ to be one of them. Therefore, among all the sides of the given $C(U)$, at least two of them are boundary leaves of $K_{i}$. This implies that $n \leq \frac{12 g-12+4 b}{2}=6 g-6+2 b$. Assume that $\lambda_{1}, \ldots, \lambda_{r} \in A_{\Lambda}, 0 \leq r<n$, are isolated leaves and $\lambda_{r+1}, \ldots, \lambda_{n} \in A_{\Lambda}$ are isolated from one side. Every $\bar{\lambda}_{s}$ with $s>r$ belongs to a unique minimal infinite sublamination $\Lambda_{s}^{\prime}$ of $\Lambda$.

Now the maximal chain of sublaminations $\Lambda_{k} \varsubsetneqq \Lambda_{k-1} \varsubsetneqq \ldots \varsubsetneqq \Lambda_{1} \varsubsetneqq \Lambda_{0}=\Lambda$ of $\Lambda$ is constructed as follows: We pass from $\Lambda_{i-1}$ to $\Lambda_{i}$ by removing all the isolated open geodesics $\lambda_{i} \in A_{\Lambda}$ with $i \leq r$, and then every minimal infinite sublamination $\Lambda_{t}^{\prime}$ of $\Lambda$ and every closed geodesic $c_{j} \in A_{\Lambda}$. Obviously the length of $\Lambda$ is less than $9 g-9+3 b$. This finishes the proof of Claim 5 .

Now, from Lemma 3.7 the homeomorphism $f$ preserves the length of a maximal lamination $\Lambda$, and this finishes the proof of (1).

Now we prove (2). First, we claim that if $K$ is a geodesic lamination consisting of $k$ closed geodesics, then $f(K)$ is a finite lamination. To prove this, we consider a maximal finite geodesic lamination $\Lambda$ containing a generalized pair of pants decomposition $P$ with $K \subset P$. By (1), $f(\Lambda)$ is a maximal finite lamination, and thus our claim follows. From this claim and Lemma 3.7 it follows that if $K_{1}=\{c\}$ is a geodesic lamination consisting of a single closed geodesic, then the image of $K_{1}$ by $f$ consists of a single closed geodesic.

Now we have proved statement (2) for $n=1$ and we proceed by induction. We assume that the statement is true for all $n \leq k$ and we prove it for $n=k+1$. 
Assume that $K$ consists of $k+1$ closed geodesics. If our statement were not true for $n=k+1$, then from our inductive assumption and Lemma 3.7. $f(K)$ would consist of $k$ closed geodesics, say $c_{1}, \ldots, c_{k}$, plus one open geodesic. Then, any sublamination of $K$ consisting of $k$ geodesics would be sent to $\left\{c_{1}, \ldots, c_{k}\right\}$. But this is impossible since $f$ is a homeomorphism. This completes the induction and the proof of (2).

Now we prove (3). Let $K$ be a finite lamination. Obviously, a minimal sublamination of $K$ consists of a single closed geodesic. Assume that $f(K)$ is not finite. Then there is an infinite minimal sublamination $\Lambda_{0}$ of $f(K)$. From Lemma 3.5, $\Lambda_{0}$ is the image of a minimal sublamination $K_{0}$ of $K$, by $f$, i.e. $f\left(K_{0}\right)=\Lambda_{0}$. But from $(2), f\left(K_{0}\right)$ consists of a single closed geodesic. Therefore we have a contradiction, which proves (3).

\section{Proof of Theorem 1.1}

We first prove that if $f: \mathcal{G L}(S) \rightarrow \mathcal{G L}(S)$ is a homeomorphism with respect to the topology $\mathcal{T}$, then there is a homeomorphism $h: S \rightarrow S$ such that $h_{*}=f$.

We need the following lemma.

Lemma 4.1. Assume that $\left(\Lambda_{n}\right)$ is a sequence of laminations that converges to a lamination $\Lambda$ with respect to the Hausdorff topology. Assume also that $\left(\Lambda_{n}\right)$ converges to $\Lambda^{\prime}$ with respect to the Thurston topology. Then $\Lambda^{\prime} \subset \Lambda$.

Proof. If $\Lambda^{\prime}$ is not contained in $\Lambda$, then there exists a point $x \in \Lambda^{\prime}-\Lambda$. Therefore there exists an open ball $B(x, 2 \varepsilon)$ in $S$ of center $x$ and radius $2 \varepsilon$ such that $B(x, 2 \varepsilon) \cap$ $\Lambda=\emptyset$. We also have $\Lambda^{\prime} \in \mathcal{U}_{B(x, \varepsilon)}$.

Now, since $\Lambda_{n} \rightarrow \Lambda$ with respect to the Hausdorff topology we deduce that there is $n_{0} \in \mathbb{N}$ such that the following holds:

$$
\Lambda_{n} \cap B(x, \varepsilon)=\emptyset \forall n \geq n_{0} .
$$

On the other hand, $\Lambda_{n} \rightarrow \Lambda^{\prime}$ with respect to the Thurston topology. This implies that there exists $n_{1} \in \mathbb{N}$ such that $\Lambda_{n} \in \mathcal{U}_{B(x, \varepsilon)}$ for $n \geq n_{1}$ and hence $\Lambda_{n} \cap B(x, \varepsilon) \neq$ $\emptyset$ for $n \geq n_{1}$. This gives a contradiction to the relation (2) which implies that $\Lambda^{\prime} \subset \Lambda$.

Now note that there is a natural identification between the subset $\mathcal{C G} \mathcal{L}(S)$ and the complex of curves $\mathcal{C}(S)$ of $S$. Therefore, from Proposition 3.8, $f$ induces an automorphism on $\mathcal{C}(S)$. From [4, 5] and [6] and under the hypothesis of Theorem 1.1 . we obtain a homeomorphism $h: S \rightarrow S$ such that $h_{*}=f$ on $\mathcal{C G} \mathcal{L}(S)$.

We now prove that $h_{*}=f$ on $\mathcal{F} \mathcal{G} \mathcal{L}(S)$. Composing $f$ with $\left(h_{*}\right)^{-1}$, if necessary, it suffices to assume that $f=$ id on $\mathcal{C G} \mathcal{L}(S)$ and prove that $f=$ id on $\mathcal{F} \mathcal{G L}(S)$. To do it, we first prove two lemmas:

Lemma 4.2. Let $\Lambda=\left\{\gamma, \gamma_{1}, \gamma_{2}\right\}$ be a geodesic lamination consisting of two disjoint simple closed geodesics $\gamma_{1}, \gamma_{2}$ and one open geodesic $\gamma$ spiraling in one direction about $\gamma_{1}$ and in the other direction about $\gamma_{2}$. Then $f(\Lambda)=\Lambda$.

Proof. We take a generalized pair of pants decomposition $P$ and a maximal finite geodesic lamination $\Lambda_{P}$ such that

(1) $\Lambda \subset \Lambda_{P}$;

(2) for each component $R$ of $S-P$ and every pair $\lambda_{1} \neq \lambda_{2}$ of boundary geodesics of $R$ there is a leaf $\lambda$ of $\Lambda_{P}$ contained in $R$ which spirals in one direction about $\lambda_{1}$ and in the other direction about $\lambda_{2}$; 
(3) for every component $\gamma$ of $P$ the leaves of $\Lambda_{P}$ spiraling about $\gamma$ from different sides of $\gamma$ induce opposite orientations on $\gamma$.

Such a lamination $\Lambda_{P}$ can be approximated in the Hausdorff topology and hence in the Thurston topology by a sequence $C_{n}$ of simple closed geodesics, where the $C_{n}$ are viewed as elements of $\mathcal{C G} \mathcal{L}(S)$. This implies that $f\left(\Lambda_{P}\right)=\Lambda_{P}$. Indeed, $C_{n} \rightarrow \Lambda_{P}$ with respect to the Hausdorff topology and hence with respect to the Thurston topology. Therefore $f\left(C_{n}\right) \rightarrow f\left(\Lambda_{P}\right)$ with respect to the Thurston topology. This implies, since $f$ is the identity on $\mathcal{C} \mathcal{G} \mathcal{L}(S)$, that $C_{n} \rightarrow f\left(\Lambda_{P}\right)$ with respect to the Thurston topology. From Lemma 4.1 we deduce that $f\left(\Lambda_{P}\right) \subset \Lambda_{P}$. But from Lemma 3.7 the laminations $\Lambda_{P}$ and $f\left(\Lambda_{P}\right)$ have the same length. Therefore $f\left(\Lambda_{P}\right)$ cannot have a smaller number of leaves than $\Lambda_{P}$. Therefore $f\left(\Lambda_{P}\right)=\Lambda_{P}$.

Let us now set $f(\Lambda)=\Lambda^{\prime}$. From Proposition 3.8 and Corollary 3.4 we deduce that $\Lambda^{\prime}=\left\{\gamma^{\prime}, \gamma_{1}, \gamma_{2}\right\}$, where $\gamma^{\prime}$ spirals about $\gamma_{1}$ and about $\gamma_{2}$. We shall prove that $\gamma^{\prime}=\gamma$.

We can find a surface $Q \subset S$ with geodesic boundary containing the lamination $\Lambda$ and which is the closure of a component of $S-P$ such that $Q$ is one of the following types:

(i) a torus with one boundary component, with $\partial Q=\gamma_{1}$ or $\partial Q=\gamma_{2}$;

(ii) a pair of pants with no cusps, with $\gamma_{1} \cup \gamma_{2} \subset \partial Q$;

(iii) a generalized pair of pants with a single cusp with $\partial Q=\gamma_{1} \cup \gamma_{2}$.

First we show that $\gamma^{\prime} \subset Q$. Let $P_{\gamma}=P \cup\{\gamma\} \subset \Lambda_{P}$. Then it is not hard to show, using Corollary 3.4 and Proposition 3.8, that $f\left(P_{\gamma}\right)=P \cup \gamma^{\prime}$. Therefore $P \cap\left\{\gamma^{\prime}\right\}=\emptyset$. This implies that if $\gamma^{\prime}$ is not contained in $Q$, then it is contained in some generalized pair of pants $Q^{\prime}$ with $\gamma_{1}, \gamma_{2} \in \partial Q^{\prime}$ and $\operatorname{Int}(Q) \cap \operatorname{Int}\left(Q^{\prime}\right)=\emptyset$. Thus, we may find a simple closed geodesic $\gamma_{0}$ of $S$ which intersects only one of the geodesics $\gamma, \gamma^{\prime}$ and $\gamma_{0} \cap \gamma_{i}=\emptyset$ for $i=1,2$. To prove the last statement we need the assumption on the topological type of $S$ that we made in the introduction. Assume without loss of generality that $\gamma \cap \gamma_{0}=\emptyset$ and $\gamma^{\prime} \cap \gamma_{0} \neq \emptyset$. From Corollary 3.4 the lamination $\Lambda_{1}=\left\{\gamma, \gamma_{1}, \gamma_{2}, \gamma_{0}\right\}$ is sent to a lamination $\Lambda_{1}^{\prime}$ which should contain the geodesics $\gamma^{\prime}, \gamma_{1}, \gamma_{2}, \gamma_{0}$. But this is impossible since $\gamma^{\prime} \cap \gamma_{0} \neq \emptyset$, and hence $Q^{\prime}=Q$.

Now in the cases (ii) and (iii) above there is a unique leaf of $\Lambda_{P}$ contained in $Q$ that spirals about $\gamma_{1}$ and $\gamma_{2}$, namely the leaf $\gamma$. This implies that $\gamma^{\prime}=\gamma$ since $f(\Lambda)=\Lambda^{\prime} \subset \Lambda_{P}=f\left(\Lambda_{P}\right)$ and $\gamma^{\prime} \subset Q$.

Now consider the case (i); without loss of generality we assume that $\partial Q=\gamma_{2}$. In this case, the lamination $\Lambda_{P}$ which contains $\Lambda$ and which satisfies the requirements (1)-(3) above satisfies $\Lambda_{P} \cap Q=\left\{\gamma_{1}, \gamma_{2}, \gamma, \delta_{1}, \delta_{2}\right\}$, where $\gamma$ is a geodesic spiraling about $\gamma_{1}$ in both directions but from different sides of $\gamma_{1}$ and $\delta_{1}, \delta_{2}$ are spiraling about $\gamma_{1}$ and $\gamma_{2}$. Denote by $\Lambda_{Q}$ the lamination $\Lambda_{P} \cap Q$.

Also, if $\gamma$ is an open simple geodesic of $S$, we denote by $\bar{\gamma}$ the closure of $\gamma$ in $S$. Obviously $\bar{\gamma}$ is a geodesic lamination and consists of $\gamma$ and two or one additional simple closed geodesics on which $\gamma$ is spiraling. We note that we can talk about $f(\{\bar{\gamma}\})$ and not about $f(\{\gamma\})$, since $\bar{\gamma}$ is a lamination but $\gamma$ is not.

Claim 1. $f\left(\Lambda_{Q}\right)=\Lambda_{Q}$ and $f(\{\bar{\gamma}\})=\{\bar{\gamma}\}$.

Proof of Claim 1. Since $f\left(\Lambda_{P}\right)=\Lambda_{P}$ and $f$ is the identity on $\mathcal{C G} \mathcal{L}(S)$ we deduce that $f\left(\Lambda_{Q}\right)=\Lambda_{Q}$. On the other hand, $f(\{\bar{\gamma}\})=\{\bar{\gamma}\}$ since $\gamma$ is the unique leaf of $\Lambda_{Q}$ spiraling about $\gamma_{1}$ in both directions.

Now let $\beta_{1}$ be a simple open geodesic in $Q$ spiraling in both directions about $\gamma_{1}$ from the same side of $\gamma_{1}$ and such that $\beta_{1} \cap \delta_{1}=\emptyset$. 
Claim 2. $f\left(\left\{\overline{\beta_{1}}\right\}\right)=\left\{\overline{\beta_{1}}\right\}$.

Proof of Claim 2. Since $f(\{\bar{\gamma}\})=\{\bar{\gamma}\}$, it is easy to prove that either $f\left(\left\{\overline{\beta_{1}}\right\}\right)=$ $\left\{\overline{\beta_{1}}\right\}$ or $f\left(\left\{\overline{\beta_{1}}\right\}\right)=\left\{\overline{\beta_{2}}\right\}$, where $\beta_{2}$ is the unique geodesic in $Q$ spiraling about $\gamma_{1}$ in both directions and from the same side of $\gamma_{1}$ and such that $\beta_{2} \cap \gamma=\emptyset$. Now, we may find an open geodesic $\alpha$, as well as a closed geodesic $\gamma_{1}^{\prime}$, such that:

$\gamma_{1}^{\prime} \cap Q=\emptyset$

$\alpha$ is spiraling about $\gamma_{1}$ and $\gamma_{1}^{\prime}$;

$\alpha \cap \beta_{1}=\emptyset$ but $\alpha \cap \beta_{2} \neq \emptyset$.

From (ii) we have that $f(\{\bar{\alpha}\})=\{\bar{\alpha}\}$, and therefore we deduce that $f\left(\left\{\overline{\beta_{1}}\right\}\right)=\left\{\overline{\beta_{1}}\right\}$, and Claim 2 is proved.

We are now able to prove that $f\left(\left\{\overline{\delta_{i}}\right\}\right)=\left\{\overline{\delta_{i}}\right\}, i=1,2$. Indeed, considering the lamination $\Lambda_{Q}$ it is clear from Claim 1 that $f\left(\left\{\gamma_{1}, \gamma_{2}, \delta_{1}, \delta_{2}\right\}\right)=\left\{\gamma_{1}, \gamma_{2}, \delta_{1}, \delta_{2}\right\}$. Now we consider the lamination $\Lambda_{0}=\left\{\gamma_{1}, \gamma_{2}, \beta_{1}, \delta_{1}\right\}$. Since $f\left(\Lambda_{0}\right) \subset Q, f\left(\left\{\overline{\beta_{1}}\right\}\right)=\left\{\overline{\beta_{1}}\right\}$ and $\beta_{1} \cap \delta_{2} \neq \emptyset$ we easily deduce that $f\left(\left\{\overline{\delta_{1}}\right\}\right)=\left\{\overline{\delta_{1}}\right\}$, and this completes the proof of Lemma 4.2 .

Lemma 4.3. Let $\Lambda=\left\{\gamma, \gamma_{1}\right\}$ be the geodesic lamination consisting of one simple closed geodesic $\gamma_{1}$ and one open geodesic $\gamma$ spiraling in both directions about $\gamma_{1}$. Then $f(\Lambda)=\Lambda$.

Proof. First we find a surface $Q \subset S$ with geodesic boundary containing $\gamma$ and $\gamma_{1}$ such that $Q$ is one of the following surfaces:

(i) a torus with one boundary component such that $\gamma_{1}$ is not the boundary of $Q$;

(ii) a torus with one boundary component such that $\gamma_{1}=\partial Q$;

(iii) a generalized pair of pants with cusps such that $\gamma_{1} \subset \partial Q$.

In case (i) we distinguish the following three subcases:

$\left(i_{a}\right) \gamma$ spirals in both directions about $\gamma_{1}$ from different sides of $\gamma_{1}$ and induces opposite orientation on $\gamma_{1}$ from each side;

$\left(i_{b}\right) \gamma$ spirals in both directions about $\gamma_{1}$ from the same side of $\gamma_{1}$;

$\left(\mathrm{i}_{c}\right) \gamma$ spirals in both directions about $\gamma_{1}$ from different sides of $\gamma_{1}$ and induces the same orientation on $\gamma_{1}$ from each side.

Then, as in the proof of Lemma 4.2 we prove that $\gamma \subset Q$.

Assume that $f(\Lambda)=\Lambda^{\prime}$. From Proposition 3.8 and Corollary 3.4, we deduce that $\Lambda^{\prime}=\left\{\gamma^{\prime}, \gamma_{1}\right\}$, where $\gamma^{\prime}$ spirals in both directions about $\gamma_{1}$.

In case (iii) we proceed as in Lemma4.2 More precisely, we consider a lamination $\Lambda_{P}$ which satisfies requirements (1)-(3) of the proof of Lemma 4.2 and can check that there is a unique leaf of $\Lambda_{P}$ contained in $Q$, namely the leaf $\gamma$, which spirals about $\gamma_{1}$. As in Lemma 4.2, this implies that $\gamma^{\prime}=\gamma$. Therefore, $\Lambda=\Lambda^{\prime}$.

In case (ii) we consider a simple closed geodesic $\gamma_{2}$ in $Q$ such that $\gamma_{2} \cap \gamma=\emptyset$. We also consider a simple open geodesic $\delta$ in $Q$ spiraling about $\gamma_{1}$ in one direction and about $\gamma_{2}$ in the other direction and such that $\left\{\gamma_{1}, \gamma_{2}, \gamma, \delta\right\}$ is a geodesic lamination. Then, from Lemma 4.2 we have that $f(\{\bar{\delta}\})=\{\bar{\delta}\}$, which implies that $f(\{\bar{\gamma}\})=$ $\{\bar{\gamma}\}$.

Cases $\left(i_{a}\right)$ and $\left(i_{b}\right)$ have been studied respectively in Claims 1 and 2 above. In each case we proved that $f(\{\bar{\gamma}\})=\{\bar{\gamma}\}$.

In case $\left(\mathrm{i}_{c}\right)$ we consider the lamination $\left\{\gamma_{1}, \gamma_{2}, \gamma, \delta_{1}, \delta_{2}\right\}$, where $\delta_{1}$ and $\delta_{2}$ are geodesics in $Q$ spiraling both in one direction about $\gamma_{1}$ and in the other direction about $\gamma_{2}=\partial Q$. Now, it is easy to see that $f(\{\bar{\gamma}\})$ is a lamination consisting of 
$\gamma_{1}$ and one open leaf spiraling in both directions about $\gamma_{1}$. On the other hand, from Lemma 4.2 we have that $f\left(\left\{\overline{\delta_{i}}\right\}\right)=\left\{\overline{\delta_{i}}\right\}, i=1,2$. Hence we deduce that $f(\{\bar{\gamma}\})=\{\bar{\gamma}\}$, and the lemma is proved.

Now we can prove that $f=$ id on $\mathcal{F} \mathcal{G} \mathcal{L}(S)$. Indeed, consider $K \in \mathcal{F} \mathcal{G} \mathcal{L}(S)$ such that $f(K)=K^{\prime} \neq K$. Then, without loss of generality, we may assume that there is a leaf $\gamma$ of $K$ such that $\gamma$ is not a leaf of $K^{\prime}$. First we remark that $\gamma$ cannot be a closed geodesic since, if $\gamma$ is closed, $f(\{\gamma\})=\{\gamma\}$ and by Corollary 3.4 $f(\{\gamma\})=\{\gamma\} \subset f(K)$. Let $\gamma$ be an open geodesic and assume that $\gamma$ spirals about two disjoint closed geodesics $\gamma_{1}, \gamma_{2}$. Consider the lamination $\left\{\gamma, \gamma_{1}, \gamma_{2}\right\}$. By Lemma 4.2. $f\left(\left\{\gamma, \gamma_{1}, \gamma_{2}\right\}\right)=\left\{\gamma, \gamma_{1}, \gamma_{2}\right\}$, and hence $\left\{\gamma, \gamma_{1}, \gamma_{2}\right\} \subset f(K)$ by Corollary 3.4. Therefore $\gamma$ is a leaf of $f(K)$, a contradiction which implies that $f(K)=K$. (The case where $\gamma$ spirals about a single closed geodesic $\gamma_{1}$ in both directions is treated similarly using Lemma 4.3.)

Finally we will show that $f=$ id on $\mathcal{G} \mathcal{L}(S)$. Let $\Lambda \in \mathcal{G} \mathcal{L}(S)$. Then from Proposition 2.6 such a lamination $\Lambda$ can be approximated in the Hausdorff topology and hence in the Thurston topology by a sequence $F_{n}$ of finite laminations. This implies that $f(\Lambda)=\Lambda$. Indeed, $F_{n} \rightarrow \Lambda$ with respect to the Hausdorff and hence with respect to the Thurston topology. Therefore $f\left(F_{n}\right)=F_{n} \rightarrow f(\Lambda)$ with respect to the Thurston topology. From Lemma 4.1 we deduce that $f(\Lambda) \subset \Lambda$. Both $\Lambda$ and $f(\Lambda)$ are infinite laminations. Therefore, from Theorem 2.4 (III), $\Lambda$ and $f(\Lambda)$ consist of the disjoint union of a finite number of infinite minimal sublaminations with a finite set of isolated, open geodesics, each end of which spirals onto a minimal sublamination. Now, if $f(\Lambda) \neq \Lambda$, then $f(\Lambda)$ must consist either of a smaller number of minimal sublaminations of $\Lambda$ or/and of a smaller number of isolated, open geodesics. This implies that length $(f(\Lambda))<\operatorname{length}(\Lambda)$, which contradicts Lemma 3.7. Therefore $f(\Lambda)=\Lambda$.

This proves that any homeomorphism $f: \mathcal{G L}(S) \rightarrow \mathcal{G L}(S)$ with respect to the topology $\mathcal{T}$ is induced by a homeomorphism $h: S \rightarrow S$.

Thus, the natural homomorphism from the extended mapping class group $\Gamma^{*}(S)$ of $S$ to the group of homeomorphisms of the space $\mathcal{G} \mathcal{L}(S)$, equipped with the Thurston topology, is an isomorphism. To complete the proof of Theorem 1.1, we need to show that if two elements of $\Gamma^{*}(S)$ have the same action on $\mathcal{G} \mathcal{L}(S)$, then they are equal. Under the hypothesis of the theorem and furthermore if we exclude the case of a closed surface of genus 2, this result follows from the fact that the homomorphism from $\Gamma^{*}(S)$ to the automorphism group of the complex of curves $C(S)$ of $S$ is injective (see [4]). It remains to consider the case where $S$ is a closed surface of genus 2 .

Thus, we now assume that $S$ is a closed surface of genus 2. It is known that in this case if $g$ is an element of $\Gamma^{*}(S)$ that induces the identity map on $C(S)$, then $g$ is either the identity element or a hyperelliptic involution. It remains to show that a hyperelliptic involution does not induce the identity on $\mathcal{G} \mathcal{L}(S)$. To see this, let $\iota$ denote the hyperelliptic involution. We note that there is a pair of pants decomposition $P$ of $S$ that is invariant by $\iota$. By using various ways in which an infinite geodesic in a pair of pants spirals along the boundary components, we can complete the three curves in $P$ into a maximal geodesic lamination $\Lambda$ whose image by $\iota$ is a lamination that is different from $\Lambda$. This shows that $\iota$ does not induce the identity map on $\mathcal{G L}(S)$. This completes the proof of Theorem 1.1 


\section{ACKNOWLEDGEMENTS}

The authors would like to thank Ken'ichi Ohshika, who read a preliminary version of this paper and corrected several mistakes, and Yi Huang, who pointed out a gap in one of the arguments in a previous version. The authors would also like to thank the anonymous referee for help in improving the text and for correcting a mistake in the proof of Lemma 2.5.

\section{REFERENCES}

[1] Herbert Busemann, The geometry of geodesics, Academic Press Inc., New York, NY, 1955. MR.0075623 (17,779a)

[2] R. D. Canary, D. B. A. Epstein, and P. L. Green, Notes on notes of Thurston [MR0903850], Fundamentals of hyperbolic geometry: selected expositions, with a new foreword by Canary, London Math. Soc. Lecture Note Ser., vol. 328, Cambridge Univ. Press, Cambridge, 2006, pp. 1-115. MR2235710

[3] Andrew J. Casson and Steven A. Bleiler, Automorphisms of surfaces after Nielsen and Thurston, London Mathematical Society Student Texts, vol. 9, Cambridge University Press, Cambridge, 1988. MR964685 (89k:57025)

[4] N. V. Ivanov, Automorphisms of Teichmüller modular groups, Topology and geometryRohlin Seminar, Lecture Notes in Math., vol. 1346, Springer, Berlin, 1988, pp. 199-270, DOI 10.1007/BFb0082778. MR970079 (89m:57012)

[5] Mustafa Korkmaz, Automorphisms of complexes of curves on punctured spheres and on punctured tori, Topology Appl. 95 (1999), no. 2, 85-111, DOI 10.1016/S0166-8641(97)002782. MR1696431 (2000d:57025)

[6] Feng Luo, Automorphisms of the complex of curves, Topology 39 (2000), no. 2, 283-298, DOI 10.1016/S0040-9383(99)00008-7. MR1722024(2000j:57045)

[7] Katsuhiko Matsuzaki and Masahiko Taniguchi, Hyperbolic manifolds and Kleinian groups, Oxford Mathematical Monographs, The Clarendon Press, Oxford University Press, New York, 1998. MR 1638795 (99g:30055)

[8] K. Ohshika, Reduced Bers boundaries of Teichmüller spaces, to appear in Ann. Inst. Fourier.

[9] K. Ohshika, A note on the rigidity of unmeasured lamination space, Proceedings of the AMS 141 (2013), no. 12, 4385-4389. MR3105880

[10] J. P. Otal, Le théorème d'hyperbolisation pour les variétés fibrées de dimension 3, Soc. Math. France, Astérisque 235, 1996. MR.1402300 (97e:57013)

[11] Athanase Papadopoulos, A rigidity theorem for the mapping class group action on the space of unmeasured foliations on a surface, Proc. Amer. Math. Soc. 136 (2008), no. 12, 4453-4460, DOI 10.1090/S0002-9939-08-09433-1. MR2431062 (2009e:57031)

[12] W. Thurston, The geometry and topology of three manifolds, Princeton Lecture Notes, 1979.

Laboratory of Mathematics, Agricultural University of Athens, Iera Odos 75, 118 55 Athens, Greece

E-mail address: bakis@aua.gr

Laboratory of Mathematics, Agricultural University of Athens, Iera Odos 75, 118

55 Athens, Greece

E-mail address: papadoperakis@aua.gr

Institut de Recherche Mathématique Avancée, Université de Strasbourg and CNRS,

7 rue René Descartes, 67084 Strasbourg Cedex, France

E-mail address: athanase.papadopoulos@math.unistra.fr 